\title{
Evaluación de la ingesta calórica en pacientes diabéticos tipo 2 descontrolados, en Lima Metropolitana, durante el año 2011
}

\author{
Clever Arias, Marco Núñez, Miriam Inga, Felipe Sosa, Oscar Acosta \\ Departamento Académico de Ciencias Dinámicas, Facultad de Medicina, UNMSM
}

Objetivos: Determinación de la TMB y GET en pacientes diabéticos tipo 2, durante el año 2011.

Diseño: Observacional con sistema de medición, descriptivo.

Institución: Departamento Académico de Ciencias Dinámicas, Facultad de Medicina, UNMSM.

Participantes: Pacientes con diabetes mellitus tipo 2.

Intervenciones: En 300 pacientes con diabetes mellitus tipo 2 se realizó encuestas socio- económicas, medidas antropométricas, evaluación de la tasa metabólica basal (TMB) y gasto energético total (GET) de los pacientes diabéticos tipo 2; posterior a ello se tomó una muestra de sangre para los análisis bioquímicos.

Principales medidas de resultados: Peso, talla, IMC, TMB, GET, glucosa mg\%.

Resultados: El 58\% de los pacientes presentó obesidad de primer y segundo grado y $17,6 \%$ sobrepeso; $64,7 \%$ no desarrollaba dieta y presentó hiperglucemia. El $60 \%$ de los pacientes presentó TMB muy por debajo de lo normal e hiperglicemia. El 56\% de los pacientes presentó GET muy bajo en relación con su elevada ingesta calórica; en ellos se apreció obesidad de primer y segundo grado. El $59 \%$ de los pacientes no desarrollaba actividad física y presentó hiperglucemia.

Conclusiones: Al tener un $64,7 \%$ de los pacientes que no desarrollaba dieta adecuada y $59 \%$ de estos sin desarrollar actividad física, un $56 \%$ de los mismos presentó un GET muy por debajo de lo requerido y $60 \%$ una TMB baja, Io cual puede estar provocando un aumento en la hiperglicemia que presenta dicha población.

Palabras clave: Diabetes mellitus, tasa metabólica basal (TMB), gasto energético total (GET).

\section{Características del crecimiento y desarrollo fetal en hiperémesis gravídica}

Gloria Larrabure, Humberto Farfán, Ronal Aragón, Víctor Cruz Instituto Nacional Materno Perinatal

Introducción: La hiperémesis gravídica (HG) cursa con restricción del crecimiento intrauterino (RCIU) y peso bajo al nacer.

Objetivos: Determinar en gestante con HG las curvas de crecimiento fetal por ecografía (CCF) y peso al nacer (PAN).

Diseño: Descriptivo, prospectivo.

Institución: Hospitalización y consultorio de endocrinología, Instituto Nacional Materno Perinatal (INMP).

Participantes: Gestantes con HG y sus recién nacidos (RN).

Intervenciones: En los recién nacidos (RN) de 45 gestantes con diagnóstico de HG, se obtuvo antropometría del RN (peso, talla) y CCF. Se consideró RCIU al peso fetal estimado por biometría por debajo del percentil 10 en la curva de crecimiento fetal; macrosomía, al peso al nacer de 4 kilos o mayor; peso bajo al nacer, al peso menor de 2,5 kilos.

Principales medidas de resultados: Características de gestantes y de los recién nacidos.

Resultados: Promedios maternos: edad gestacional 10,7 semanas, edad 24,5 años, ganancia de peso durante la gestación 12,2 kg, parto a las 38,8 semanas. Promedios de los RN: peso $3430,5 \mathrm{~g}$ (dos macrosómicos), talla 50,2 cm. Nacimiento de los niños en Lima 66,7\%, sierra 22,3\%, costa no Lima 6,6\%, selva 4,4\%; parto en el INMP $31,1 \%(14)$; primigestas $57 \%$, secundigestas $28,5 \%$ tercigestas $14,5 \%$, parto eutócico $50 \%$ (nueve RN femeninos, cinco masculinos), Ápgar a los 5 minutos $92 \%$. No hubo peso menor de $2500 \mathrm{~g}$ ni RCIU.

Conclusiones: La hiperémesis gravídica en el Instituto Nacional Materno Perinatal no se relacionó con peso bajo al nacer o con restricción del crecimiento intrauterino. Hubo 2 macrosómicos.

Palabras clave: Hiperémesis gravídica, restricción del crecimiento intrauterino, curva de crecimiento fetal por ecografía. 